\title{
Structure of a $\beta_{1}$-adrenergic $G$ protein-coupled receptor
}

\author{
Tony Warne, Maria J. Serrano-Vega, Jillian G. Baker", Rouslan Moukhametzianov, Patricia \\ C. Edwards, Richard Henderson, Andrew G.W. Leslie, Christopher G. Tate ${ }^{\star}$, and Gebhard \\ F.X. Schertler \\ MRC Laboratory of Molecular Biology, Hills Road, Cambridge CB2 OQH, UK \\ \#Institute of Cell Signalling, Medical School, Queen's Medical Centre, University of Nottingham \\ NG7 2UH, UK
}

\section{Summary}

G protein-coupled receptors play a major role in transmembrane signalling in higher organisms and many are important drug targets. We report the $2.7 \AA$ resolution crystal structure of a $\beta_{1^{-}}$ adrenergic receptor in complex with the high-affinity antagonist cyanopindolol. The modified turkey receptor had been selected to be in its antagonist conformation and its thermostability improved by earlier limited mutagenesis. The ligand-binding pocket comprises 15 side chains from amino acid residues in 4 transmembrane a-helices and extracellular loop 2. This loop defines the entrance of the ligand-binding pocket and is stabilised by two disulphide bonds and a sodium ion. Cyanopindolol binding to the $\beta_{1}$-adrenergic receptor and carazolol binding to the $\beta_{2^{-}}$ adrenergic receptor involve similar interactions. A short well-defined helix in cytoplasmic loop 2, not observed in either rhodopsin or the $\beta_{2}$-adrenergic receptor, directly interacts via a tyrosine with the highly conserved DRY motif at the end of helix 3 that is essential for receptor activation.

G protein-coupled receptors (GPCRs) are a large family of integral membrane proteins that are prevalent in eukaryotes from yeast to man, and which function as key intermediaries in the transduction of signals from outside to inside the cell1. Activating molecules (agonists), such as hormones and neurotransmitters, bind to GPCRs from the extracellular side of the cell membrane and induce a large conformational change which propagates to the cytoplasmic surface 2,3 , resulting in activation of $\mathrm{G}$ proteins and a consequent change in the level of intracellular messengers such as cAMP, $\mathrm{Ca}^{2+}$ or signalling lipids. There are over 800 different human GPCRs4, all sharing the characteristic arrangement of 7 transmembrane a-helices with the polypeptide $\mathrm{N}$-terminus on the extracellular side of the plasma membrane5. Analysis of their primary amino acid sequences has resulted in the definition of a number of families6, the largest of which, family A, includes the archetypal GPCR, rhodopsin. The three human $\beta$-adrenergic receptor ( $\beta$ AR) subtypes, $\beta_{1}, \beta_{2}$ and $\beta_{3}$, belong to family A and share $51 \%$ sequence identity between $\operatorname{Trp}^{1.31}-\mathrm{Asp}^{5.73}$ and Glu ${ }^{6.30}$ $\mathrm{Cys}^{\mathrm{H} 8-\mathrm{Cterm}}$ i.e. excluding the $\mathrm{N}$ - and $\mathrm{C}$-termini and most of cytoplasmic loop 3 (Supplementary Fig 1; superscripts refer to Ballesteros-Weinstein numbering7). Drugs that

\footnotetext{
*Joint corresponding authors MRC Laboratory of Molecular Biology, Hills Road, Cambridge CB2 0QH, UK cgt@ mrclmb.cam.ac.ukgfx@mrc-lmb.cam.ac.uk Telephone +44-(0)1223-402338 +44-(0)1223-402328 Fax +44-(0)1223-213556. Author contributions. TW devised and carried out receptor expression, purification, crystallisation and cryo-cooling of the crystals. Receptor stabilisation and baculovirus expression were performed by MJSV; both authors were also involved in data collection and preliminary crystallographic analyses of the crystals. PE helped with the crystal cryo-cooling strategy and in diffraction data collection. JGB performed the functional cAMP and reporter gene assays. RM was involved in data collection and processing. AGWL processed the final data, solved and refined the structure and assisted with manuscript preparation. The overall project management and manuscript preparation was by RH, CGT and GFXS.
}

Conflicts of interest GFXS, CGT and RH are all on the Scientific Advisory Board of Heptares Therapeutics Ltd.

Author information. Co-ordinates and structure factors have been submitted to the PDB database under accession code 2VT4. 
inhibit $\beta_{1}$ and $\beta_{2}$ receptor signalling (antagonists and inverse agonists) are used to modulate heart function and are known as $\beta$-blockers8, but selective $\beta_{1}$-antagonists are preferred since they have fewer side effects due to bronchial constriction via $\beta_{2}$ receptors in the lung. In contrast to the $\beta_{1}$ and $\beta_{2}$ receptors, the $\beta_{3}$-adrenergic receptor $\left(\beta_{3} \mathrm{AR}\right)$ is found in adipose tissue, where adrenaline stimulates metabolism, and is a potential target to treat obesity. Elucidation of the specificity determinants for drug affinity of the different $\beta A R$ subtypes will allow the development of better subtype-specific $\beta$-blockers, with fewer side effects.

A milestone in the study of $\beta$ ARs was recently reached with the publication of a $\beta_{2^{-}}$ adrenergic receptor $\left(\beta_{2} \mathrm{AR}\right)$ structure in complex with an antibody fragment, $\beta_{2} \mathrm{AR}: F a b 9$, followed by the higher resolution structure of an engineered $\beta_{2} \mathrm{AR}$ fused in the middle of the third cytoplasmic loop (CL3) to T4 lysozyme, $\beta_{2}$ AR-T410. These structures, both containing the high affinity antagonist carazolol, defined the overall architecture of $\beta_{2} \mathrm{AR}$, and the structure of the ligand-binding pocket. However, the structures also raised questions of how a range of compounds can bind to the different but closely related $\beta$ AR subtypes with different affinities. For example, the human $\beta_{1}$ and $\beta_{2}$ receptors are $67 \%$ identical within their transmembrane regions, but the residues that directly surround the ligand binding pocket appear to be identical. Despite these similarities, larger antagonists such as CGP 20712A (see Supplementary Fig. 2) bind 500 times more strongly to $\beta_{1}$ AR than to $\beta_{2} \mathrm{AR}$, whilst ICI 118551 shows a 550 fold specificity for $\beta_{2} \mathrm{AR}$ over $\beta_{1} \mathrm{AR} 11$. There are also $\beta_{1}$ and $\beta_{2}$ specific agonists12. As an important step towards understanding subtype specificity, we have determined the structure of a $\beta_{1}$-adrenergic receptor $\left(\beta_{1} \mathrm{AR}\right)$.

\section{Crystallisation of $\beta_{1} A R$}

GPCR crystallisation is challenging, since GPCRs are usually unstable in detergent, contain unstructured regions, and spontaneously cycle between an inactive antagonist state (R) and an active agonist state $\left(R^{*}\right)$, which may further decrease the stability 13 . The human $\beta_{1} A R$ is more difficult to purify than $\beta_{2} \mathrm{AR}$, because it is very unstable in detergent. We therefore used turkey (Meleagris gallopavo) $\beta_{1} \mathrm{AR}$, which is more stable than human $\beta_{1} \mathrm{AR} 14$ though less stable than human $\beta_{2} \mathrm{AR}$ (MJSV and CGT, unpublished observation). A mutated receptor, $\beta_{1} \mathrm{AR}-\mathrm{m} 23$, was constructed with enhanced thermostability over the wild-type receptor of $21^{\circ} \mathrm{C}$ and an altered equilibrium between $\mathrm{R}$ and $\mathrm{R}^{*}$ so that the mutant receptor was preferentially in the antagonist (R) state 15 . The receptor construct, $\beta_{1}$ AR36-m23 (Fig. 1) purified in octylthioglucoside and in the presence of cyanopindolol gave good crystals showing isotropic diffraction beyond $2.7 \AA$.

\section{Pharmacological analysis of $\beta_{1} A R-m 23$}

The mutant receptor $\beta_{1} \mathrm{AR}-\mathrm{m} 23$ bound the antagonists dihydroalprenolol and cyanopindolol with similar affinities to the wild-type receptor, but the agonists noradrenaline and isoprenaline bound more weakly by a factor of 2470 and 650 respectively 15 . This reflects a change in the $\mathrm{R}$ to $\mathrm{R}^{*}$ equilibrium of the receptor towards the antagonist $\mathrm{R}$ state. From this we predicted that, in a $\mathrm{G}$ protein-coupling assay, the receptor would show no basal activity and that the concentration of agonist required for signalling would be orders of magnitude higher. Signalling assays were performed on stable cell lines expressing the wild type $\beta_{1} \mathrm{AR}$ truncated at the $\mathrm{N}$ - and $\mathrm{C}$ - termini ( $\beta_{1}$ ARtrunc) and also containing the six thermostabilising mutations (m23) (Supplementary Fig. 3). $\beta_{1}$ ARtrunc-m23 coupled efficiently to G proteins and elicited a robust stimulation of cAMP-responsive reporter gene, although the agonist concentration response curve, as expected was shifted to the right16. The drug ICI 118551, an inverse agonist for both $\beta_{1} \mathrm{AR} 17$ and $\beta_{2} \mathrm{AR} 18$, showed no reduction in the basal level of cAMP when added to cells containing $\beta_{1}$ ARtrunc-m23, at a concentration 100-fold above its $\mathrm{K}_{\mathrm{i}}$, implying there is negligible basal constitutive activity. The structure we have determined 
contains the very high affinity antagonist cyanopindolol in the binding pocket and represents closely the inactive conformation with respect to $\mathrm{G}$ protein coupling.

\section{Overall structure and the extracellular loops}

The structure was solved by molecular replacement to $2.7 \AA$ resolution with an $\mathrm{R}_{\text {work }}$ of 0.212 and $R_{\text {free }}$ of 0.268 (Supplementary Table 1). The four receptor molecules in the unit cell, labelled A-D (Supplementary Figs. 4-6), were all very similar except that molecules A and D both had a $60^{\circ}$ kink in helix $1(\mathrm{H} 1)$. Also modelled are 31 water molecules, $4 \mathrm{Na}^{+}$ ions and 14 detergent molecules (see Supplementary Information). Unless otherwise stated, all further discussion refers to molecule $\mathrm{B}$, as this molecule has an unkinked $\mathrm{H} 1$ and a relatively well-ordered H8. The helix boundaries, disordered regions and overall structural motifs are presented in Fig. 1.

The amino acid sequence of turkey $\beta_{1}$ AR19 is $82 \%$ and $67 \%$ identical to human $\beta_{1}$ AR and human $\beta_{2}$ AR, respectively, over residues Trp $40^{1.31}$-Asp $242^{5.73}$ and Glu285 2.30

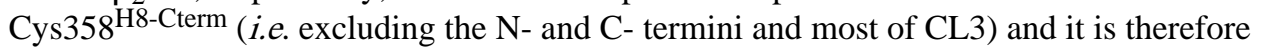
expected that the structure of the transmembrane regions of $\beta_{1} \mathrm{AR}$ and $\beta_{2} \mathrm{AR}$ should be very similar. Our superposition of $\beta_{2} \mathrm{AR}$ (PDB code $2 \mathrm{RH} 1$ ) and $\beta_{1} \mathrm{AR}$ (chain $\mathrm{B}$ ) is based on selected residues in H3, H5, H6 and H7 since we were particularly interested in comparing the ligand binding pockets; 78 alpha carbons can be superimposed with an rmsd of $0.25 \AA$. The rmsd over all transmembrane helices is $0.7 \AA$ ( $269 C_{\alpha}$ atoms; Supplementary Fig 7). Comparison of the $\beta_{1} \mathrm{AR}$ and $\beta_{2} \mathrm{AR}$ structures reveals no evidence for any significant changes in backbone conformation at the sites of the six point mutants introduced 15 to stabilise $\beta_{1} \mathrm{AR}$. This is consistent with the observation that $\beta_{1} \mathrm{AR}-\mathrm{m} 23$ binds antagonists with similar affinities to the wild type receptor 15 and that it can couple efficiently to $G$ proteins, though at higher agonist concentration (Supplementary Fig. S3). The basis for the thermostabilisation by the six mutations $\mathrm{R} 68^{1.59} \mathrm{~S}, \mathrm{M} 90^{2.53} \mathrm{~V}, \mathrm{Y} 227^{5.58} \mathrm{~A}, \mathrm{~A} 282^{6.27} \mathrm{~L}$, $\mathrm{F} 327^{7.37} \mathrm{~A}$ and $\mathrm{F} 338^{7.48} \mathrm{M}$ is not immediately apparent from the structure.

The structures of the three extracellular loops (EL1-3) in $\beta_{1}$ AR are very similar to those of $\beta_{2} \mathrm{AR}\left(\mathrm{C}_{\mathrm{a}}\right.$ rmsd $\left.0.8 \AA\right)$, consistent with the high sequence conservation of these regions in the $\beta$ AR family (Supplementary Fig. 1). On the extracellular surface, a clear peak in the electron density is present at a position co-ordinated by the carbonyl groups of residues Cys192, Asp195, Cys198 and a water molecule (Supplementary Fig 8). This density was assigned to a sodium ion based on its coordination geometry20. Its role, bound at the negative end of the EL2 a-helix dipole, may be to stabilise the helical conformation of EL2 and thus the structure of the entrance to the ligand-binding pocket. The large difference in EL2 conformation between the $a$-helix found in $\beta_{2} \mathrm{AR}$ and the $\beta$-hairpin that closes off the retinal binding site in rhodopsin is confirmed in the structure of $\beta_{1} \mathrm{AR}$, suggesting that the a-helix may be a common feature in GPCRs that bind their ligands rapidly and reversibly.

\section{Cytoplasmic loop structure}

In all GPCRs, CL2 and CL3 are believed to play an important role in the binding, selectivity and activation of G proteins, CL2 being important for the strength of the interaction and CL3 for specificity $21,22,23,24,25$. The $\beta_{1} \mathrm{AR}$ and $\beta_{2} \mathrm{AR}$ structures, along with rhodopsin 26 , have similar conformations for CL1, but there are major differences in CL2 and CL3. The CL3 differences are not of physiological relevance because they arise from deletions $\left(\beta_{1} \mathrm{AR}\right)$, deletion and insertion of T4 lysozyme ( $\left.\beta_{2} \mathrm{AR}-\mathrm{T} 4\right)$ or formation of an antibody complex ( $\left.\beta_{2} \mathrm{AR}: \mathrm{Fab}\right)$, with only the rhodopsin structure having a native CL326. However, differences in the conformation of CL2 (Fig. 3) are important, because this region is very highly conserved between $\beta_{1} \mathrm{AR}$ and $\beta_{2} \mathrm{AR}$, though poorly conserved with rhodopsin. In $\beta_{1}$ AR, CL2 forms a short $\alpha$-helix (residues Pro146 $6^{3.57}$-Leu152 $2^{3.63}$; Supplementary Fig. 9) 
parallel to the membrane surface whereas in both $\beta_{2} \mathrm{AR}$ structures and in rhodopsin this loop is in an extended conformation (Fig. 2). This short a-helix cannot be accommodated in either the $\beta_{2} \mathrm{AR}: \mathrm{Fab}$ complex9 or the $\beta_{2} \mathrm{AR}-\mathrm{T} 4$ fusion 10 crystal structures perhaps because of lattice contacts with adjacent molecules. In $\beta_{1} A R, C L 2$ also makes lattice contacts, but these are different between each of the four molecules and it is therefore likely that the helical conformation found here represents the physiologically relevant structure for all $\beta$ ARs in the inactive conformation.

The CL2 loop has been proposed to function as the switch enabling $\mathrm{G}$ protein activation 21 and it is clear from the $\beta_{1} A R$ structure that this short $a$-helix interacts directly with the highly conserved Asp $138^{3.49} \mathrm{Arg} 139^{3.50}$ Tyr140 3.51 (DRY) motif in H3. Tyr149 in CL2 is located sufficiently close to Asp $138^{3.49}$ to allow the formation of a hydrogen bond (Fig. 2) between the tyrosine hydroxyl and the aspartate side chain. Supporting evidence for this structural role of Tyr149 comes from the observation that the Y149A mutation makes $\beta_{1} \mathrm{AR}$ less thermally stable (Supplementary Table 2). The equivalent Tyr141 in both $\beta_{2} \mathrm{AR}$ structures is in a cavity between $\mathrm{H} 3, \mathrm{H} 4$ and $\mathrm{H} 6$, but the biological relevance of this is unclear, due to the perturbations in this region caused by either the T4 lysozyme fusion or by the bound antibody. Interestingly, a pattern of mutations consistent with an a-helical conformation for CL2 was found in the muscarinic M5 receptor and the equivalent M5 mutation Y138A led to increased constitutive activity21. Thus it is likely that both the tyrosine residue and the CL2 a-helix play key roles in G protein coupling.

A salt bridge between $\mathrm{Arg}^{3.50}$ and Glu ${ }^{6.30}$, termed the "ionic lock", (Fig. 2) was proposed to play an essential role in maintaining GPCRs in an inactive state27 but to break upon receptor activation. Since the $\beta_{1}$ AR structure represents a receptor lacking basal activity and complexed with an antagonist, it is highly likely to represent the $\mathrm{R}$ conformation. Yet this salt bridge is not present either in the $\beta_{1} \mathrm{AR}$ or the $\beta_{2} \mathrm{AR}$ structures (Fig. 2). This suggests that the ionic lock is not an essential feature of the inactive state. Even in dark-state rhodopsin, where these two charged residues are within hydrogen bonding distance $26,28,29$, the side chain B-factors of the two residues differ greatly $\left(20-40 \AA^{2}\right) 26$ so there is no direct experimental evidence for any "lock".

\section{Selectivity of the ligand binding pocket}

Cyanopindolol is similar in structure to carazolol (Supplementary Fig. 2) that is present in the ligand binding pocket of both $\beta_{2}$ AR structures; both ligands bind with very high affinity to all $\beta$ ARs. In the $\beta_{1}$ AR structure there are 15 amino acid residues, using a $3.9 \AA$ distance criterion, whose side chains make contacts with cyanopindolol in the ligand binding pocket; 4 side chains are from H3, 3 from H5, 4 from H6, 2 from H7 and 2 from EL2 (Fig. 3). All these residues are identical to those in human $\beta_{2} \mathrm{AR}$ and the mode of binding of cyanopindolol to $\beta_{1} \mathrm{AR}$ is, therefore, similar to that of carazolol in $\beta_{2} \mathrm{AR}$. However, the extra ring in the carazolol heterocyclic ring, due to van der Waals contact with Tyr199.38 in $\beta_{2} \mathrm{AR}$, pushes the ligand more deeply into the binding site. The nitrogen in the cyano-moiety of cyanopindolol makes a weak hydrogen bond with the hydroxyl of Thr2035.34, which is located together with Phe201 5.32 on EL2 (Fig. 3). The same hydrogen bonds between the ligand and Asp $121^{3.32}$, Asn $329^{7.39}$ and Ser21 $1^{5.42}$ are present in both $\beta_{1} A R$ and $\beta_{2} A R$ structures, but the side chain rotamer conformation of Ser211 $1^{5.42}$ is different (Fig 4 and Supplementary Data).

To explain why some ligands preferentially bind to either $\beta_{1} \mathrm{AR}$ or $\beta_{2} \mathrm{AR}$, which is important in understanding the sub-type specificity of the human receptors 11 , there must be differences in amino acid residues close to the ligand binding pocket that directly or indirectly affect binding. A comparison of residues within $8 \AA$ of the binding pocket 
identified only two residues that are different between human $\beta_{1} A R$ and $\beta_{2} A R$ subtypes. The respective residues are Val1724.56 and Phe $325^{7.35}$ in $\beta_{1} \mathrm{AR}$, equivalent to Thr164 1.56 and Tyr308 7.35 in $\beta_{2}$ AR. These differences introduce polar residues near the binding pocket of $\beta_{2} \mathrm{AR}$ relative to $\beta_{1} \mathrm{AR}$ (Fig. 4), which could affect ligand selectivity. Tyr3087.35 has also been implied by mutagenesis studies30,31 to be important for agonist selectivity in $\beta_{2}$ AR. In $\beta_{2} \mathrm{AR}, \mathrm{Tyr} 308^{7.35}$ is positioned close to the binding pocket and can form a hydrogen bond to Asn293 ${ }^{6.55}$. In $\beta_{1}$ AR the side chain of Asn $310^{6.55}$ is closer to the cyano group of cyanopindolol and the equivalent residue, Phe $325^{7.35}$, is further from the binding pocket (Fig. 4). As a result, there is no contact between Phe $325^{7.35}$ in $\beta_{1}$ AR and cyanopindolol.

Part of the ligand binding site is formed by EL2. Although the backbone positions within this highly structured region of $\beta_{1}$ AR differ from $\beta_{2}$ AR by an rmsd of only $0.84 \AA$, compared with $0.63 \AA$ between the same residues in molA and molB, there are significant amino acid side chain differences in this region. These change the shape and charge distribution around the entrance to the ligand binding pocket (Supplementary Fig. 10) with an ion pair formed between Asp192 $2^{5.31}$ and Lys305 7.32 in $\beta_{2}$ AR that is absent in $\beta_{1} A R$ because the respective residues are both aspartate (Asp200 ${ }^{5.31}$ and Asp322 $2^{7.32}$ ). Differences between $\beta_{1} \mathrm{AR}$ and $\beta_{2} \mathrm{AR}$ in this region could affect ligand binding especially for larger ligands with extensions that make direct interactions with non-conserved side chains. Recent mutational studies show that EL2 influences the specificity of ligand binding to both the normal (orthosteric) site 32,33 and the sites of allosteric modulators 34 , and that the loop flexibility is important to the binding kinetics35.

The structure of $\beta_{1} \mathrm{AR}$, when compared to $\beta_{2} \mathrm{AR}$, provides a sound basis for studying selectivity differences between $\beta A R$ antagonists structurally similar to cyanopindolol and carazolol. However, many ligands, such as the inverse agonist CGP 20712A (Supplementary Fig. 2), show very high selectivities 11 but are physically larger and structurally distinct from either cyanopindolol or carazolol. These ligands could well make contact with residues other than those described here.

\section{Agonist binding and GPCR activation}

The $\beta_{1}$ AR crystal structure shows the inactive state of the receptor but it is notable that many agonists, including the natural ligands adrenaline and noradrenaline are smaller than many of the best antagonists, including cyanopindolol. Agonists have a shorter distance, by two carbon-carbon bonds or 2-3 $\AA$, between the catechol hydroxyl groups or their equivalent and the obligatory amine nitrogen. We superimposed (Fig. 4B) a model of adrenaline with that of cyanopindolol and examined its relationship to the side chains of Asp $121^{3.32}$ and Asn $329^{7.39}$, which make hydrogen bonds with the amine, and those of Ser2 $11^{5.42}$, Ser $212^{5.43}$ and Ser215 5.46 , which are expected to hydrogen bond with the meta- and parahydroxyl groups on the catechol ring36,37,38. As noticed by Rosenbaum et al.39, the catechol hydroxyl groups are well spaced and well oriented to interact with the side chain hydroxyl groups of Ser2 $11^{5.42} / \mathrm{Ser} 212^{5.43}$ and Ser215 $5^{5.46}$ on H5, but cannot reach far enough to make good hydrogen bonds if the amine occupies the same position as it does adjacent to Asp1213.32 in the cyanopindolol complex, without a substantial structural change in the receptor. It seems very reasonable that the ligand binding site in $\beta_{1} \mathrm{AR}$ will contract by $2-3 \AA$ on activation so that both ends of adrenaline can make good interactions with the residues on $\mathrm{H} 3 / \mathrm{H} 7$ and $\mathrm{H} 5$. This view is also supported by engineered zinc binding sites that activate the receptor40,41. How this tightening around the ligand binding site could propagate to the cytoplasmic surface and cause an outward 5-6 $\AA$ movement of H62,3 is difficult to predict, since all the transmembrane helices except $\mathrm{H} 1$ and $\mathrm{H} 3$ have pronounced kinks at conserved proline residues, which means they could easily bend. However, one speculation is that the pulling of $\mathrm{H} 5$ towards the centre of the receptor on activation could 
force $\mathrm{H} 3$ and H6 apart, causing cytoplasmic loops CL2 and CL3 to move apart as observed in photoactivated rhodopsin 3 and trigger recruitment of $\mathrm{G}$ protein complex.

\section{Methods Summary \\ Purification and crystallisation}

The $\beta_{1}$ AR construct T34-424/His642 was the starting point for the generation of the $\beta_{1}$ AR36-m23 construct that crystallized. The C-terminus was further truncated after Leu367, and 6 histidines were added. Two segments, comprising residues 244-271 and 277-278 of CL3, were also deleted. The construct included the following 8 point mutations: $\mathrm{C} 116^{3.27} \mathrm{~L}$ increased expression; $\mathrm{C} 358 \mathrm{~A}$ at the $\mathrm{C}$-terminus of $\mathrm{H} 8$ removed palmitoylation and helped crystallisation; R68 ${ }^{1.59} \mathrm{~S}, \mathrm{M} 90^{2.53} \mathrm{~V}, \mathrm{Y} 227^{5.58} \mathrm{~A}, \mathrm{~A} 282^{6.27} \mathrm{~L}, \mathrm{~F} 327^{7.37} \mathrm{~A}$ and $\mathrm{F} 338^{7.48} \mathrm{M}$ thermostabilised the receptor in the antagonist conformation15. Baculovirus expression and receptor purification 42 were performed in decylmaltoside, with the detergent exchanged to octylthioglucoside on the alprenolol sepharose column. Crystals were obtained by vapour diffusion at $18^{\circ} \mathrm{C}$ with hanging drops after addition of an equal volume of reservoir solution, 0.1M N-(2-acetamido)iminodiacetic acid: $\mathrm{NaOH}$ pH 6.9-7.3 and 29-32\% PEG600 to receptor concentrated to $6.0 \mathrm{mg} / \mathrm{ml}$.

\section{Data collection, structure solution and refinement}

Diffraction data were collected from many crystals on beamlines ID13 and ID23-2 at ESRF, Grenoble43,44; the data used for structure determination were collected at ID23-2 with a 10 $\mu \mathrm{m}$ beam using three positions on a single cryo-cooled crystal (100 K). Images were processed with MOSFLM and SCALA45. The structure was solved by molecular replacement with PHASER46, using the structure of human $\beta_{2}$ AR10 as an initial model. All four copies of the molecule in the triclinic unit cell were located (Supplementary Figs. 4 and 5). The amino acid sequence was corrected, the model refined with PHENIX47 and rebuilt with $\mathrm{O} 48$ (see Full Methods online for further details). An overview of the B-factor distribution for $\beta_{1}$ AR molecules A and B is shown in Supplementary Fig. 6. Figures were produced using Pymol (DeLano Scientific LLC).

\section{Supplementary Material}

Refer to Web version on PubMed Central for supplementary material.

\section{Acknowledgments}

This work was supported by a joint grant from Pfizer Global Research and Development and from the MRCT Development Gap Fund to CGT and RH, in addition to core funding from MRC. GFXS was financially supported by a Human Frontier Science Project (HFSP) programme grant (RG/0052), a European Commission FP6 specific targeted research project (LSH-2003-1.1.0-1) and an ESRF long-term proposal. JGB is funded by a Wellcome Trust Clinician Scientist Fellowship. We thank Elliott Ross (UTSW) for his support in the initial stages of the $\beta_{1}$ AR project at the LMB and for his comments on the manuscript. In addition, we would also like to thank Reinhard Grisshammer (NIH), Ed Hulme (NIMR), Fiona Marshall (Heptares), Malcolm Weir (Heptares) and our colleagues at LMB for their comments on the manuscript. We also thank beamline staff at the European Synchrotron Radiation Facility, particularly C. Riekel and M. Burghammer at ID13 and D. Flot and S. McSweeney at ID 23-2.

\section{References}

1. Fredriksson R, Schioth HB. The repertoire of G-protein-coupled receptors in fully sequenced genomes. Mol. Pharmacol. 2005; 67:1414-1425. [PubMed: 15687224]

2. Hubbell WL, Altenbach C, Hubbell CM, Khorana HG. Rhodopsin structure, dynamics, and activation: A perspective from crystallography, site-directed spin labeling, sulfhydryl reactivity, and disulfide cross-linking. Adv. Protein Chem. 2003; 63:243-290. [PubMed: 12629973] 
3. Altenbach C, et al. High resolution distance mapping in rhodopsin reveals the pattern of helix movement due to activation. Proc. Natl. Acad. Sci. USA. 2008 In Press.

4. Foord SM, et al. International Union of Pharmacology. XLVI. G protein-coupled receptor list. Pharmaco.1 Rev. 2005; 57:279-288.

5. Baldwin JM, Schertler GF, Unger VM. An alpha-carbon template for the transmembrane helices in the rhodopsin family of G-protein-coupled receptors. J. Mol. Biol. 1997; 272:144-164. [PubMed: 9299344]

6. Bockaert J, Pin JP. Molecular tinkering of G protein-coupled receptors: an evolutionary success. EMBO J. 1999; 18:1723-1729. [PubMed: 10202136]

7. Ballesteros JA, Weinstein H. Integrated methods for the construction of three dimensional models and computational probing of structure function relations in $\mathrm{G}$ protein-coupled receptors. Methods Neurosci. 1995; 25:366-428.

8. Black JW. Drugs from Emasculated Hormones - the Principle of Syntopic Antagonism (Nobel Lecture). Angew. Chem. Int. Edit. 1989; 28:886-894.

9. Rasmussen SG, et al. Crystal structure of the human beta2 adrenergic G-protein-coupled receptor. Nature. 2007; 450:383-387. [PubMed: 17952055]

10. Cherezov V, et al. High-resolution crystal structure of an engineered human beta2-adrenergic G protein-coupled receptor. Science. 2007; 318:1258-1265. [PubMed: 17962520]

11. Baker JG. The selectivity of beta-adrenoceptor antagonists at the human beta1, beta 2 and beta3 adrenoceptors. Br. J. Pharmacol. 2005; 144:317-322. [PubMed: 15655528]

12. Sugimoto $Y$, et al. beta(1)-selective agonist (-)-1-(3,4-dimethoxyphenetylamino)-3-(3,4dihydroxy)-2-propanol [(-)-RO363] differentially interacts with key amino acids responsible for beta(1)-selective binding in resting and active states. J. Pharmacol. Exp. Ther. 2002; 301:51-58. [PubMed: 11907156]

13. Gether U, et al. Structural instability of a constitutively active G protein-coupled receptor. Agonistindependent activation due to conformational flexibility. J. Biol. Chem. 1997; 272:2587-2590. [PubMed: 9006889]

14. Parker EM, Kameyama K, Higashijima T, Ross EM. Reconstitutively active G protein-coupled receptors purified from baculovirus-infected insect cells. J. Biol. Chem. 1991; 266:519-527. [PubMed: 1845979]

15. Serrano-Vega MJ, Magnani F, Shibata Y, Tate CG. Conformational thermostabilization of the beta1-adrenergic receptor in a detergent-resistant form. Proc. Natl. Acad. Sci. U S A. 2008; 105:877-882. [PubMed: 18192400]

16. Baker JG. Site of action of beta-ligands at the human beta 1-adrenoceptor. J. Pharmacol. Exp. Ther. 2005; 313:1163-1171. [PubMed: 15716385]

17. Lattion A, Abuin L, Nenniger-Tosato M, Cotecchia S. Constitutively active mutants of the beta1adrenergic receptor. FEBS Lett. 1999; 457:302-306. [PubMed: 10471797]

18. Samama P, et al. Negative Antagonists Promote an Inactive Conformation of the Beta(2)Adrenergic Receptor. Mol. Pharmacol. 1994; 45:390-394. [PubMed: 7908404]

19. Yarden Y, et al. The avian beta-adrenergic receptor: primary structure and membrane topology. Proc. Natl. Acad. Sci. U S A. 1986; 83:6795-6799. [PubMed: 3018746]

20. Harding MM. Metal-ligand geometry relevant to proteins and in proteins: sodium and potassium. Acta Cryst. D. 2002; 58:872-874. [PubMed: 11976508]

21. Burstein ES, Spalding TA, Brann MR. The second intracellular loop of the m5 muscarinic receptor is the switch which enables G-protein coupling. J. Biol. Chem. 1998; 273:24322-24327. [PubMed: 9733718]

22. Wong SKF, Parker EM, Ross EM. Chimeric Muscarinic Cholinergic Beta-Adrenergic Receptors That Activate Gs in Response to Muscarinic Agonists. J. Biol. Chem. 1990; 265:6219-6224. [PubMed: 2156845]

23. Wong SKF, Ross EM. Chimeric Muscarinic Cholinergic - Beta-Adrenergic Receptors That Are Functionally Promiscuous among G-Proteins. J. Biol. Chem. 1994; 269:18968-18976. [PubMed: 8034654] 
24. Wess J, Bonner TI, Dorje F, Brann MR. Delineation of Muscarinic Receptor Domains Conferring Selectivity of Coupling to Guanine Nucleotide-Binding Proteins and 2nd Messengers. Mol. Pharmacol. 1990; 38:517-523. [PubMed: 2172767]

25. Scarselli M, Li B, Kim SK, Wess J. Multiple residues in the second extracellular loop are critical for M3 muscarinic acetylcholine receptor activation. J. Biol. Chem. 2007; 282:7385-7396. [PubMed: 17213190]

26. Li J, et al. Structure of bovine rhodopsin in a trigonal crystal form. J. Mol. Biol. 2004; 343:14091438. [PubMed: 15491621]

27. Ballesteros JA, et al. Activation of the beta 2-adrenergic receptor involves disruption of an ionic lock between the cytoplasmic ends of transmembrane segments 3 and 6. J. Biol. Chem. 2001; 276:29171-29177. [PubMed: 11375997]

28. Palczewski K, et al. Crystal structure of rhodopsin: A G protein-coupled receptor. Science. 2000; 289:739-745. [PubMed: 10926528]

29. Okada T, et al. The retinal conformation and its environment in rhodopsin in light of a new 2.2 angstrom crystal structure. J. Mol. Biol. 2004; 342:571-583. [PubMed: 15327956]

30. Kikkawa H, Isogaya M, Nagao T, Kurose H. The role of the seventh transmembrane region in high affinity binding of a beta(2)-selective agonist TA-2005. Mol. Pharmacol. 1998; 53:128-134. [PubMed: 9443940]

31. Isogaya $\mathrm{M}$, et al. Identification of a key amino acid of the beta2-adrenergic receptor for high affinity binding of salmeterol. Mol. Pharmacol. 1998; 54:616-622. [PubMed: 9765503]

32. Shi L, Javitch JA. The second extracellular loop of the dopamine D2 receptor lines the binding-site crevice. Proc. Natl. Acad. Sci. USA. 2004; 101:440-445. [PubMed: 14704269]

33. Klco JM, Wiegand CB, Narzinski K, Baranski TJ. Essential role for the second extracellular loop in C5a receptor activation. Nat. Struct. Mol. Biol. 2005; 12:320-326. [PubMed: 15768031]

34. Voigtlander U, et al. Allosteric site on muscarinic acetylcholine receptors: Identification of two amino acids in the muscarinic M-2 receptor that account entirely for the M-2/M-5 subtype selectivities of some structurally diverse allosteric ligands in N-methylscopolamine-occupied receptors. Mol. Pharmacol. 2003; 64:21-31. [PubMed: 12815157]

35. Avlani VA, et al. Critical role for the second extracellular loop in the binding of both orthosteric and allosteric G protein-coupled receptor ligands. J. Biol. Chem. 2007; 282:25677-25686. [PubMed: 17591774]

36. Sato T, Kobayashi H, Nagao T, Kurose H. Ser(203) as well as Ser(204) and Ser(207) in fifth transmembrane domain of the human beta(2)-adrenoceptor contributes to agonist binding and receptor activation. Br. J. Pharmacol. 1999; 128:272-274. [PubMed: 10510435]

37. Strader CD, et al. Identification of 2 Serine Residues Involved in Agonist Activation of the BetaAdrenergic-Receptor. J. Biol. Chem. 1989; 264:13572-13578. [PubMed: 2547766]

38. Liapakis G, et al. The forgotten serine - A critical role for Ser-203(5.42) in ligand binding to and activation of the beta(2)-adrenergic receptor. J. Biol. Chem. 2000; 275:37779-37788. [PubMed: 10964911]

39. Rosenbaum DM, et al. GPCR engineering yields high-resolution structural insights into beta2adrenergic receptor function. Science. 2007; 318:1266-1273. [PubMed: 17962519]

40. Elling CE, Thirstrup K, Holst B, Schwartz TW. Conversion of agonist site to metal-ion chelator site in the beta(2)-adrenergic receptor. Proc. Natl. Acad. Sci. USA. 1999; 96:12322-12327. [PubMed: 10535920]

41. Schwartz TW, et al. Molecular mechanism of 7TM receptor activation - A global toggle switch model. Annu. Rev. Pharmacol. Toxicol. 2006; 46:481-519. [PubMed: 16402913]

42. Warne T, Chirnside J, Schertler GF. Expression and purification of truncated, non-glycosylated turkey beta-adrenergic receptors for crystallization. Biochim. Biophys. Acta. 2003; 1610:133-140. [PubMed: 12586387]

43. Riekel C, Burghammer M, Schertler G. Protein crystallography microdiffraction. Curr. Opin. Struct. Biol. 2005; 15:556-562. [PubMed: 16168633]

44. Standfuss J, et al. Crystal structure of a thermally stable rhodopsin mutant. J. Mol. Biol. 2007; 372:1179-1188. [PubMed: 17825322] 
45. Evans P. Scaling and assessment of data quality. Acta Cryst. D. 2006; 62:72-82. [PubMed: 16369096]

46. McCoy AJ, et al. Phaser crystallographic software. J. Appl. Cryst. 2007; 40:658-674. [PubMed: 19461840]

47. Adams PD, et al. PHENIX: building new software for automated crystallographic structure determination. Acta Cryst. D. 2002; 58:1948-1954. [PubMed: 12393927]

48. Jones TA, Zou JY, Cowan SW, Kjeldgaard M. Improved Methods for Building Protein Models in Electron-Density Maps and the Location of Errors in These Models. Acta Crystallogr A. 1991; 47:110-119. [PubMed: 2025413]

49. Kabasch W, Sander G. Dictionary of protein secondary structure: pattern recognition of hydrogenbonded and geometriical features. Biopolymers. 1983; 22:2577-2637. [PubMed: 6667333] 


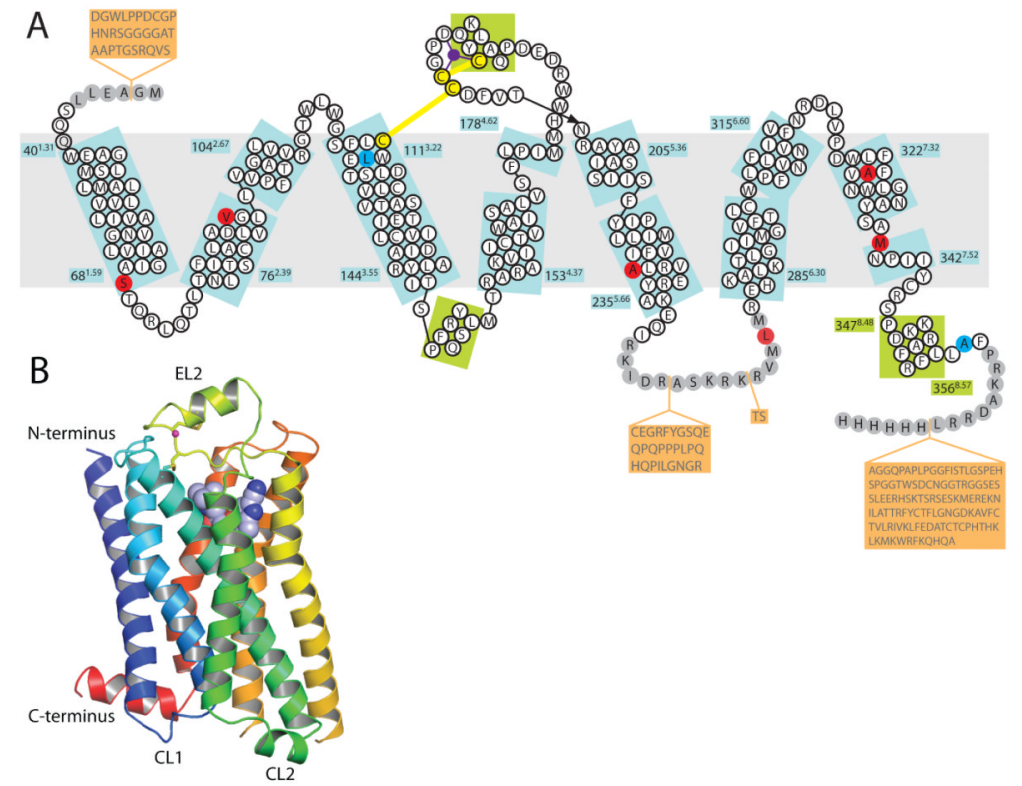

Figure 1.

Schematic representations of the turkey $\beta_{1}$ AR structure. (A) Diagram of the turkey $\beta_{1} A R$ sequence in relation to secondary structure elements. Amino sequence in white circles indicates regions that are well ordered, but sequences in grey circles were not resolved in the structure. Sequences on an orange background were deleted to make the $\beta_{1} A R$ construct for expression. Thermostabilising mutations are in red and two other mutations $\mathrm{C} 116 \mathrm{~L}$ (increases functional expression) and C358A (eliminates palmitoylation site) are in blue. The $\mathrm{Na}^{+}$ion is in purple. Numbers refer to the first and last amino acid residues in each helix (blue boxes), with the Ballesteros-Weinstein numbering in superscript. Helices were defined using the Kabasch \& Sander algorithm49, with helix distortions being defined as residues that have main chain torsion angles that differ by more than $40^{\circ}$ from standard $a$ helix values $\left(-60^{\circ},-40^{\circ}\right)$. (B) Ribbon representation of the $\beta_{1} A R$ structure in rainbow colouration (N-terminus blue, $\mathrm{C}$-terminus red), with the $\mathrm{Na}^{+}$ion in pink, the two disulphide bonds in yellow and cyanopindolol as a space-filling model. Extracellular loop 2 (EL2) and cytoplasmic loops 1 and 2 (CL1, CL2) are labelled. 

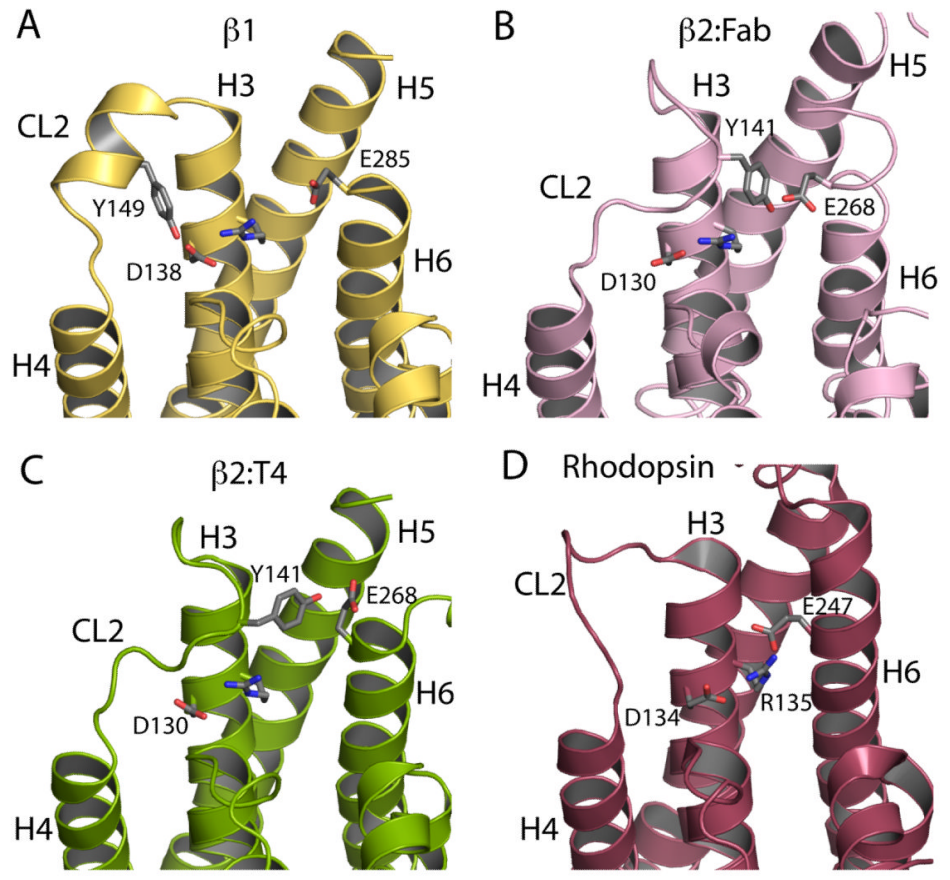

Figure 2.

Comparison of the CL2 loop regions in four GPCR structures. (A) $\beta_{1} A R$, (B) $\beta_{2} A R: T 4$ lysozyme fusion, (C) $\beta_{2}$ :Fab complex and (D) rhodopsin. Residues DR from the highly conserved conserved $\mathrm{D}^{3.48} \mathrm{R}^{3.49} \mathrm{Y}^{3.50}$ motif are shown. Residue $\mathrm{E}^{6.30}$, which is half of the putative ionic lock, is also shown as E247 in rhodopsin, and E285 and E268 in $\beta_{1}$ AR and $\beta_{2}$ AR respectively: E247 $7^{6.30}$ was thought to form a salt bridge with R135.4. but the evidence is weak. Finally, Y149 in $\beta_{1}$ forms a hydrogen bond with D138 3.49 in $\beta_{1}$ AR. 

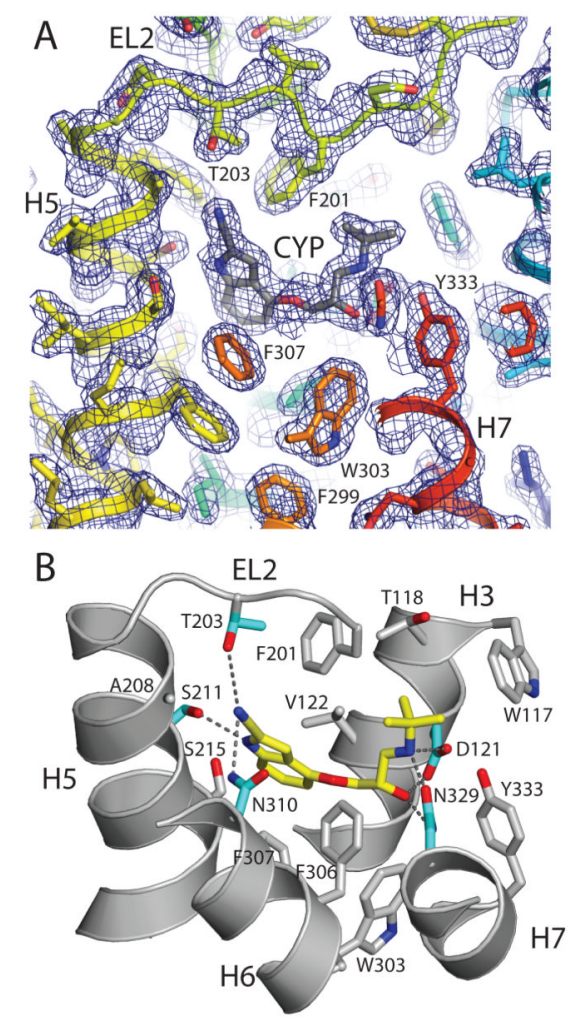

Figure 3.

Structure of the ligand binding pocket. (A) $2 \mathrm{~F}_{\mathrm{o}}-\mathrm{F}_{\mathrm{c}}$ map prior to inclusion of cyanopindolol (CYP) in the model showing the interaction of CYP with Thr203 and Phe201 in EL2. (B) Amino acid residues that interact with the ligand cyanopindolol (yellow) by polar interactions (aquamarine) or non-polar interactions (grey). 

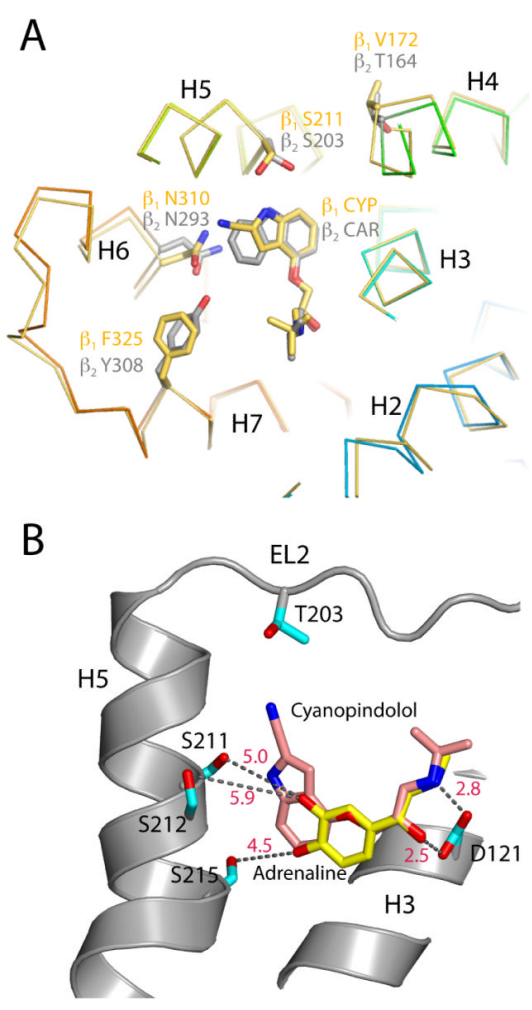

Figure 4.

Comparisons between $\beta$ receptor ligand binding pockets and the binding of different ligands. (A) Superposition of $\beta_{1} \mathrm{AR}$ molecule B with $\beta_{2} \mathrm{AR}$ (PDB code $2 \mathrm{RH} 110$ ) in the region surrounding the ligand binding site. Shown are side chains that have different rotamer conformations ( $\mathrm{N} 310^{6.55}$ and $\mathrm{S} 211^{5.42}$ ) along with two residues that are conserved yet consistently different between $\beta_{1}$ and $\beta_{2}$ receptors (F325/Y308 ${ }^{7.35}$ and V172/T164 ${ }^{4.56}$ ). Cyanopindolol (CYP) is in the ligand binding pocket of the $\beta_{1}$ receptor and carazolol (CAR) is in the $\beta_{2}$ receptor. The biggest backbone deviation is seen at the V172/T164 ${ }^{4.56}$ position. (B) Superposition of a model of the agonist, adrenaline (yellow), with the structure of the antagonist, cyanopindolol (pink), as it binds to $\beta_{1} \mathrm{AR}$, showing the distances ( $\AA$, red) to the nearest side chains known to interact with the hydoxyl groups on the catechol ring of the agonist. It is clear that a 2-3 $\AA$ tightening of the pocket around the ligand must occur on agonist binding. 